Revista Destaques Acadêmicos, Lajeado, v. 11, n. 3, 2019. ISSN 2176-3070

DOI: http://dx.doi.org/10.22410/issn.2176-3070.v11i3a2019.2294

http://www.univates.br/revistas

\title{
ASSOCIAÇÃO ENTRE PARÂMETROS NEUROMUSCULARES E CAPACIDADE FUNCIONAL EM MULHERES IDOSAS
}

\author{
Daniela Beatriz da Silva ${ }^{1}$, Jéssica Luana Dornelles da Costa², \\ Caroline Pieta Dias ${ }^{3}$, Carlos Leandro Tiggemann ${ }^{4}$
}

Resumo: O envelhecimento pode ser explicado como sendo um conjunto de processos específicos, associados à inúmeras alterações com repercussões na autonomia, funcionalidade e saúde desta população e assim, refletindo na qualidade de vida. Desta forma, o objetivo foi relacionar os parâmetros neuromusculares com a capacidade funcional de mulheres idosas. Amostra foi composta por 31 mulheres, com faixa etária entre 60 e 78 anos. A pesquisa consistiu em avaliações antropométricas, através da mensuração de massa corporal, estatura, perímetros e dobras cutâneas. A partir dos resultados destas medidas, estimou-se a massa muscular (MM) e o índice de massa muscular (IMM). A capacidade funcional foi avaliada por meio dos testes de subir degraus (DEG), sentar e levantar (CAD) e Timed Up-and-Go (TUG), sendo a força avaliada por meio do teste de uma repetição máxima (1RM) no aparelho Leg Press. Foram encontradas correlações significativas $(p<0,05)$ entre o teste de $1 R M$ com todos os testes funcionais (TUG $r=-0,533$; CAD $r=-0.380$; DEG $r=-0579$ ), porém não ocorreram correlações entre MM e a funcionalidade. Concluiu-se que neste estudo a força muscular teve influência na capacidade funcional em mulheres idosas.

Palavras-chave: Envelhecimento. Capacidade funcional. Força. Massa muscular

1 Graduada em Educação Física Bacharelado, Universidade do Vale do Taquari - Univates, Profissional de Educação Física.

2 Graduada em Educação Física Bacharelado e Licenciatura, Universidade do Vale do TaquariUnivates, Profissional de Educação Física.

3 Doutora em Ciências do Movimento Humano, Universidade Federal do Rio Grande do Sul, Docente.

4 Doutor em Ciências do Movimento Humano, Universidade do Vale do Taquari - Univates, Docente. 


\section{INTRODUÇÃO}

O envelhecimento pode ser explicado como sendo um conjunto de processos, podendo ser apresentado pela perda da capacidade de adaptação e pela diminuição da funcionalidade. Associando assim, inúmeras alterações com repercussões na mobilidade, autonomia, funcionalidade e saúde desta população, refletindo na qualidade de vida (CARVALHO; SOARES, 2004). De acordo com as estimativas, a população idosa aumenta a cada ano e, segundo o Instituto Brasileiro de Geografia e Estatística (IBGE, 2014), no Brasil, a expectativa de vida passou de 62,5 anos em 1980, para 74,9 anos em 2013, havendo um aumento de 12,4 anos nestes 33 anos.

Com o envelhecimento, a redução da massa muscular (sarcopenia) é considerada um dos principais fatores associados à perda da mobilidade funcional, dependência e aumento da fragilidade (KRAUSE et al., 2006). Além dessa redução da massa muscular (MM), o avanço da idade propicia um aumento da gordura corporal, principalmente na região abdominal e intramuscular (PÍCOLI; FIGUEIREDO; PATRIZZI, 2011).

A diminuição da força muscular influência negativamente na capacidade do indivíduo de realizar as atividades do dia a dia, comprometendo sua qualidade de vida, aumentando o risco de quedas e fraturas (SILVA et al., 2006). Além disso, alterações no sistema nervoso e reduções nas secreções hormonais, acarretam problemas na marcha e no equilíbrio do indivíduo, tornando os movimentos mais lentos e diminuindo a capacidade de reação (UNIVOVSKY, 2004).

Ao comparar mulheres jovens com mulheres idosas, observou-se que mulheres jovens obtiveram melhores resultados nos testes funcionais do que as mulheres idosas (FERREIRA et al., 2008), indicando que a idade é fator decisivo no decréscimo da funcionalidade. De acordo com o estudo feito por Mendes et al. (2016), relacionando idosas sarcopênicas com não sarcopênicas, foi observado que as sarcopênicas apresentaram menor força de preensão manual, maior tempo de execução no teste de funcionalidade e menos massa magra. Contudo, embora os exercícios físicos sejam fundamentais para a melhora da função do dia a dia em idosos, Rogatto e Gobbi (2001) compararam mulheres jovens e idosas, e constataram que, mesmo com a prática regular de exercícios físicos, com o passar dos anos, houve decréscimo da força muscular nas mulheres idosas, prejudicando assim, as atividades da vida diária.

Importante observar que parte dos estudos relacionando a sarcopenia com a funcionalidade, utilizou tanto homens como mulheres na amostra (MENDES et al., 2016), bem como, comparando mulheres com diferentes idades (FERREIRA et al., 2008; ROGATTO; GOBBI, 2001). Dessa forma, esse estudo teve como objetivo relacionar os parâmetros neuromusculares com a capacidade funcional de mulheres idosas. 


\section{PROCEDIMENTOS METODOLÓGICOS}

A pesquisa se classifica como descritiva correlacional, tendo sido aprovada pelo Comitê de Ética em Pesquisa (COEP) do Centro Universitário Univates (parecer número 1.567.120). Todas as participantes leram e assinaram o Termo de Consentimento Livre e Esclarecido (TCLE), do qual estavam cientes do estudo e concordaram em participar da pesquisa por sua livre e espontânea vontade.

A amostra foi composta por 31 mulheres com idades entre 60 e 78 anos (média de 66,65 $\pm 5,13$ anos), aparentemente saudáveis, selecionadas através de meios de comunicação, como jornal, rádio e folders sobre o estudo. A amostra foi caracterizada quanto ao nível de atividade física por meio da aplicação do Questionário Internacional de Atividade Física (IPAQ, 2005), sendo 23 (74,2\%) consideradas ativas e oito $(25,8 \%)$ consideradas sedentárias. Foram adotados como critérios de inclusão para participação do estudo, os sujeitos que: (1) não tivessem praticado treinamento de força pelo mínimo de seis meses antes do começo do estudo; (2) ausência de histórico de doenças cardiovasculares (a exceção de hipertensão arterial controlada por medicamento), endócrinas, metabólicas, neuromusculares e articulares (à exceção de quadros leves de artrite e/ou artrose); (3) não estivessem em tratamento à base de medicamentos de uso contínuo ou eventual que afetasse o equilíbrio.

Os sujeitos foram submetidos a uma fase de familiarização, seguida de uma etapa de teste e reteste de avaliações de medidas antropométricas, desempenho funcional e força, onde foram também orientados a manutenção de suas atividades diárias habituais durante esse período (TIGGEMANN et al., 2016). Cada etapa foi realizada em dois blocos: no bloco um foram realizados os testes de antropometria (ANT), subir degraus (DEG) e o teste de força máxima (1RM). No bloco dois, foram realizados os testes de sentar e levantar (CAD) e Timed Up-and-Go (TUG). Todos os testes foram realizados nas dependências do Complexo Esportivo da Univates, no turno da tarde, tendo um intervalo mínimo de 48 horas entre os mesmos. Os retestes foram feitos na semana seguinte, respeitando a mesma ordem, para que as idosas tivessem uma melhor familiarização com os protocolos. Todos os testes foram realizados por avaliadores aptos e devidamente treinados.

A ANT consistiu na mensuração das seguintes variáveis: massa corporal (balança marca Welmy; resolução de 0,1 kg), estatura (estadiômetro marca Tonelli; resolução de $0,1 \mathrm{~cm}$ ), perímetros (trena antropométrica marca Sanny; resolução de $2 \mathrm{~m}$ ) e dobras cutâneas (plicômetro marca Cescorf; resolução de $0,1 \mathrm{~mm}$ ) tricipital, da coxa e da panturrilha. Cada dobra foi mensurada duas vezes, se houvesse diferença superior ou igual a $10 \%$ entre os valores, haveria uma terceira medida e seria utilizado o valor mediano entre elas. As medidas foram coletadas em forma de circuito para impedir que os tecidos se adaptassem à pressão contínua, levando a diminuição do valor observado. Todas as medidas seguiram os protocolos do ISAK (LOPES e RIBEIRO, 2014). 
A estimativa da MM foi realizada por meio das fórmulas sugeridas por Rech (2010), a qual utiliza as variáveis estatura, idade, perímetros e dobras cutâneas do braço, da coxa e da panturrilha. Com o resultado dessa estimativa, determina-se o índice de massa muscular (IMM), sendo determinado pela razão entre massa muscular (em quilogramas) e pela estatura (em metros ao quadrado) (RECH, 2010). A partir do cálculo do IMM é possível estimar a ocorrência e o nível de sarcopenia, sendo que, valores iguais ou superiores a $6,76 \mathrm{~kg} / \mathrm{m}^{2}$ sendo considerados como sarcopenia normal, valores entre 5,76 e $6,75 \mathrm{~kg} / \mathrm{m}^{2}$ sarcopenia moderada, e quando inferior ou igual a $5,75 \mathrm{~kg} / \mathrm{m}^{2}$ sarcopenia severa.

Os testes funcionais foram realizados em duas tentativas, com intervalo de 3 minutos entre cada tentativa, sendo registrado o menor tempo (TIGGEMANN et al., 2016). O teste de DEG consistiu em os sujeitos subirem um lance de 10 degraus sem o uso do corrimão, na velocidade máxima. Cada degrau possuía $0,18 \mathrm{~m}$ de altura e 0,26 m de comprimento, onde os sujeitos deviam subir um degrau de cada vez. O tempo foi registrado a partir do primeiro contato feito no primeiro degrau e finalizado a partir do contato com o último degrau. No teste de $\mathrm{CAD}$, os sujeitos deviam levantar e sentar em uma cadeira (assento plano com $43 \mathrm{~cm}$ altura) durante cinco repetições, sendo o tempo registrado ao comando de iniciar o primeiro levantamento (com o sujeito iniciando sentado) e no final (ao sentar após o $5^{\circ}$ levantamento). Os sujeitos deviam permanecer com os braços cruzados em frente ao tórax, realizar a extensão completa de joelhos, quadris e coluna, ao levantar, e ao sentar, apoiar completamente as costas no encosto da cadeira. $O$ teste de TUG teve início a partir da posição sentada em uma cadeira ( $43 \mathrm{~cm}$ de altura, com as costas apoiadas), os sujeitos deviam levantar da mesma (sem auxílio das mãos), caminhar e contornar um cone posicionado no solo, a $3 \mathrm{~m}$ de distância, e voltar a sentar-se (com as costas apoiadas).

Para avaliação da força máxima, o teste de $1 R M$ foi realizado no aparelho Leg Press, em que o avaliado foi instruído sobre a técnica de execução do exercício, sendo que o avaliador estava atento quanto à posição correta do praticante durante o teste. Foram permitidas no máximo cinco tentativas com intervalo de três minutos entre cada uma. A primeira tentativa foi em forma de aquecimento, entre 8 à 12 repetições com carga leve. Após cada tentativa, o peso foi sendo aumentado. $O$ teste foi interrompido a partir do momento em que o avaliado não conseguiu executar mais que uma repetição, e assim, essa última carga foi determinada como a carga máxima.

Os dados foram apresentados por meio da estatística descritiva (média \pm desvio padrão). A normalidade dos dados foi testada por meio do teste de Shapiro-Wilk, sendo a correlação entre as variáveis feitas pelo teste de Correlação de Pearson. Um nível de significância de $\mathrm{p} \leq 0,05$ foi utilizado, por meio do pacote estatístico SPSS v. 20.0. 


\section{RESULTADOS}

Os dados descritivos referentes às variáveis de ANT, funcionais e força, encontram-se na Tabela 1.

Tabela 1: Estatística descritiva (mínima, máxima, média e desvio padrão) das variáveis

\begin{tabular}{|c|c|c|c|}
\hline Variáveis $(\mathrm{n}=31)$ & Mínimo & Máximo & Média \pm DP \\
\hline \multicolumn{4}{|c|}{ Antropometria } \\
\hline $\mathrm{MC}(\mathrm{Kg})$ & 46,60 & 100,00 & $69,52 \pm 12,91$ \\
\hline $\mathrm{EST}(\mathrm{m})$ & 1,44 & 1,65 & $1,54 \pm 0,06$ \\
\hline $\operatorname{IMC}\left(\mathrm{Kg} / \mathrm{m}^{2}\right)$ & 19,30 & 39,56 & $29,19 \pm 5,29$ \\
\hline $\mathrm{MM}(\mathrm{kg})$ & 17,55 & 29,02 & $22,52 \pm 2,68$ \\
\hline $\operatorname{IMM}\left(\mathrm{Kg} / \mathrm{m}^{2}\right)$ & 6,72 & 11,48 & $9,46 \pm 1,03$ \\
\hline \multicolumn{4}{|c|}{ Funcionais } \\
\hline TUG (s) & 5,25 & 8,50 & $6,81 \pm 0,82$ \\
\hline $\mathrm{CAD}(\mathrm{s})$ & 9.00 & 16,50 & $11,49 \pm 1,77$ \\
\hline DEG (s) & 3,00 & 5,38 & $4,09 \pm 0,52$ \\
\hline \multicolumn{4}{|c|}{ Força } \\
\hline $1 \mathrm{RM}(\mathrm{Kg})$ & 12,00 & 77,00 & $41,90 \pm 18,65$ \\
\hline
\end{tabular}

Legenda: MC- massa corporal; EST- estatura; IMC- índice de massa muscular; MM-massa muscular; IMM- índice de massa muscular; TUG- Timed Up-and-Go; CAD-sentar e levantar; DEG- subir degraus; 1RM- 1 repetição máxima.

Na Tabela 2 encontram-se os valores de correlação entre as variáveis neuromusculares e funcionais, em que houve correlação do 1RM com todos os testes funcionais, porém, os testes funcionais não se correlacionaram com o IMM. Além disso, também foram encontradas correlações moderadas e significativas dos valores de 1RM com o IMM $(r=0,459 ; \mathrm{p}<0,05)$, da idade com $1 \mathrm{RM}(\mathrm{r}=-0,387 ; \mathrm{p}<0,05)$, com a MM $(\mathrm{r}=-0,535 ; \mathrm{p}<0,01)$ e com o IMM $(\mathrm{r}=-0,532$; $\mathrm{p}<0,01)$. Somente um sujeito foi classificado com sarcopenia moderada $(3,2 \%)$, demais todas classificadas como sarcopenia normal. 
Tabela 2: Valores de correlação entre as variáveis funcionais e neuromusculares

\begin{tabular}{ccccccc}
\hline & \multicolumn{2}{c}{ TUG } & \multicolumn{2}{c}{ CAD } & \multicolumn{2}{c}{ DEG } \\
\hline & $\mathrm{r}$ & $\mathrm{p}$ & $\mathrm{r}$ & $\mathrm{p}$ & $\mathrm{r}$ & $\mathrm{p}$ \\
\hline 1 RM & $\mathbf{- 0 , 5 3 3 ^ { * * }}$ & $\mathbf{0 , 0 0 2}$ & $\mathbf{- 0 , 3 8 0 ^ { * }}$ & $\mathbf{0 , 0 3 8}$ & $\mathbf{- 0 , 5 7 9 ^ { * * }}$ & $\mathbf{0 , 0 0 1}$ \\
\hline MM & $-0,165$ & 0,375 & $-0,187$ & 0,314 & $-0,022$ & 0,905 \\
\hline IMM & $-0,242$ & 0,189 & $-0,300$ & 0,101 & $-0,158$ & 0,395 \\
\hline
\end{tabular}

Legenda: TUG- Timed-Up-and-Go; CAD- sentar e levantar; DEG- subir degraus; 1RM-1 repetição máxima; MM- massa muscular; IMM- índice de massa muscular; nível de significância * $\mathrm{p}<0,05$ $e^{* *} p<0,01$.

\section{DISCUSSÃO}

O presente estudo teve como objetivo verificar a associação entre os parâmetros neuromusculares e a capacidade funcional das mulheres idosas, em que o principal resultado indicou que existe correlação entre a força máxima e os diferentes testes funcionais, sendo que não foram encontradas correlações significativas entre o IMM e MM com os testes funcionais.

Em relação à força muscular e capacidade funcional, em estudo realizado por Pícoli, Figueiredo e Patrizzi (2011), a força de preensão manual mostrou associação com a incapacidade funcional, em que indivíduos com menos força, apresentaram maior dificuldade ao caminhar, estando expostos a um maior risco de quedas. De acordo com este achado, Garcia et al. (2014) avaliaram 48 idosas, aplicando-se os testes de TUG e CAD para avaliação da capacidade funcional, e dinamômetro isocinético para o desempenho muscular de joelho. Como conclusão, encontram associação do desempenho muscular com a capacidade funcional.

Corroborando com estes achados, Tiggemann et al. (2016) em estudo longitudinal, tinham como objetivo comparar os efeitos do treinamento de força tradicional e treinamento de potência na capacidade funcional de mulheres idosas, submetidas à 12 semanas de treinamento. A força foi avaliada por meio do teste de 1RM nos exercícios de extensão de joelho, flexão de joelho, leg press, supino reto e remada baixa. Concluindo que os ganhos de força foram semelhantes entre os grupos e apresentaram um efeito significativo na capacidade funcional.

Em contrapartida, Pimentel et al. (2013) avaliaram a força de preensão palmar em 40 idosas, no intuito de avaliar a força muscular, e o teste de TUG para avaliar a mobilidade funcional. Neste estudo os autores não encontraram relação significativa de força com capacidade funcional. Da mesma forma, Roncato et al. (2014), não encontraram correlação significativa entre a força máxima e a capacidade funcional em 45 idosas. 
Uma possível explicação para a divergência apresentada pelos resultados dos estudos é que, alguns autores têm proposto a presença de um limiar de força máxima, ou seja, a relação de força com a funcionalidade só aconteceria em pessoas mais fracas, abaixo deste limiar (FERRUCCI et al., 1997). Estes mesmos autores observaram que a força máxima dos flexores dos quadris foi um preditor relevante na velocidade de caminhada e na duração do teste de sentar e levantar. Resultados de força máxima de membros superiores aos limiares sugeridos, não apontaram relação significativa com uma melhor execução nos testes funcionais. Apesar de Ferrucci et al. (1997) sugerirem isso, é importante ter cautela, pois está se referindo a equipamentos diferentes, e que podem não estar representando a força máxima real nos diferentes estudos.

Quanto aos resultados de MM e IMM, o presente estudo não encontrou uma correlação significativa com a capacidade funcional em mulheres idosas. Da mesma forma, no estudo de Gadelha et al. (2014) que avaliou 137 idosas, nenhuma das variáveis funcionais diferiu significativamente entre as mulheres sarcopênicas e não sarcopências. Ou seja, os autores não encontraram uma correlação direta entre sarcopenia e as variáveis funcionais.

Em estudo realizado por Garcia et al. (2015), do qual participaram 48 idosas, a capacidade funcional foi avaliada pelos testes de TUG e CAD, a MM foi estimada a partir das medidas das dobras cutâneas. O IMM foi obtido por meio da impedância bioelétrica, indicando que $62,5 \%$ das idosas apresentaram ausência de sarcopenia, não sendo encontrada correlação entre MM e a capacidade funcional. Ao contrário do presente estudo, Mendes et al. (2016) avaliaram 53 idosos, dentre estes homens e mulheres, sendo considerados 28 pessoas sarcopênicas e 25 não sarcopênicas. Todos participantes realizaram avaliação da capacidade funcional pelo teste TUG e composição corporal através da bioimpedância tetrapolar. Nesse estudo, foi encontrada uma diferença significativa no teste de funcionalidade com idosas sarcopênicas e não sarcopênicas, onde mulheres que apresentaram maior tempo no teste de funcionalidade também apresentaram menores valores de massa magra.

O fato de alguns estudos terem encontrado uma relação entre sarcopenia e capacidade funcional, e que difere do presente estudo, pode ser explicado devido aos níveis de composição corporal das idosas avaliadas serem muito similares e não sarcopênicos, onde somente uma idosa foi classificada com sarcopenia moderada. Garcia et al. (2015) explicam que o declínio da força acontece primeiro e de maneira mais acelerada quando comparada a diminuição de MM. Esse pode ser um dos motivos de não ser encontrada uma correlação significativa entre MM e capacidade funcional.

\section{CONCLUSÃO}

Conclui-se que a força muscular tem influência na capacidade funcional em mulheres idosas, embora a MM não tenha apresentado essa mesma relação. 
Como já explicado anteriormente, este fato pode ocorrer devido à presença de um limiar de força máxima, ou seja, esta relação existe em mulheres mais fracas e com maiores níveis de sarcopenia.

\section{REFERÊNCIAS}

CARVALHO, J.; SOARES, J. M. C. Envelhecimento e força muscular - breve revisão. Revista Portuguesa de Ciências do Desporto, v. 4, n. 3, p. 79-93, 2004. Disponível em: <http://www.fade.up.pt/rpcd/_arquivo/artigos_soltos/vol.4_nr.3/2.01_joana_ carvalho.pdf $>$ Acesso em: 01 de agosto de 2016.

COELHO, B. S.; Et Al. Comparação da força e capacidade funcional entre idosos praticantes de musculação, hidroginástica e não praticantes de exercícios físicos. Revista Brasileira de Geriatria e Gerontologia, v.17, n. 3, 2014. Disponível em: <http://www.scielo.br/scielo.php?script=sci_arttext\&pid=S1809-98232014000300497> Acesso em: 05 de agosto de 2016.

FERREIRA, L.; Et Al. Capacidade funcional em mulheres jovens e idosas: projeções para uma adequada prescrição de exercícios físicos. Revista da Educação Física/UEM, v.19, n. 3, 2008. Disponível em: <http://ojs.uem.br/ojs/index.php/RevEducFis/ article/view/5995> Acesso em: 03 de setembro de 2016.

FERRUCCI L.; Et Al. Departures From Linearity in the Relationship Between Measures of Muscular Strength and Physical Performance of the Lower Extremities: The Women's Health and Aging Study. Journal of Gerontology: Medicinal Sciences, v. 52, n. 5, p. 275-285, 1997.

GADELHA A. B.; Et Al. Associação entre força, sarcopenia e obesidade sarcopénica com o desempenho funcional de idosas. Revista de Motricidade, v.10, n.3, 2014. Disponível em: <http:/ / www.scielo.mec.pt/scielo.php?script=sci_arttext\&pid=S1646107X2014000300005>. Acesso em: 10 de maio de 2017.

GARCIA P. A.; Et Al. Relação da capacidade funcional, força e massa muscular de idosas com osteopenia e osteoporose. Pesquisa Original, Brasília, 2015. Disponível em: http:/ / www.scielo.br/pdf/fp/v22n2/2316-9117-fp-22-02-00126.pdf Acesso em: 10 de maio de 2017.

Instituto Brasileiro de Geografia e Estática - IBGE Disponível em: <http:/ /www. ibge.gov.br/home/estatistica/populacao/projecao_da_populacao/2014/defaul t_tab. shtm>. Acesso em 13 março 2016.

IPAQ. Guidelines for Data Processing and Analysis of the Internacional Physical Activity Questionnaire. 2005. Disponível em: <http:/www.ipaq.ki.se/> Acesso em: 11 de outubro de 2016.

KRAUZE, M. P.; Et Al. Alterações morfológicas relacionadas à idade. Revista Brasileira de Cineantropometria \& Desempenho Humano, v.8, n.2, p.73-77, 2006. Disponível em: <https:// periodicos.ufsc.br/index.php/rbcdh/article/ viewFile/3877/3294> Acesso em: 01 de agosto de 2016. 
LOPES, A. L.; RIBEIRO, G. S.. Antropometria aplicada à saúde e ao desempenho esportivo: Uma abordagem a partir da metodologia ISAK. 1. ed. Rubio: Rio de Janeiro, 2014.

MENDES, G. S. Et Al. Sarcopenia em idosos sedentários e sua relação com funcionalidade e marcadores inflamatórios (IL-6 e IL-10). Geriatrics, Gerontology and Aging, v. 10, n.1, p. 23 - 28, 2016. Disponível em: <http:/ / www.ggaging. com/details/69/en-US/sarcopenia-em-idosos-sedentarios-e-sua-relacao-comfuncionalidade-e-marcadores-inflamatorios--il-6-e-il-10>Acesso em: 05 de outubro de 2016.

PÍCOLI, T. S.; FIGUEIREDO, L. L.; PATRIZZI, L. J.. Sarcopenia e envelhecimento. Fisioterapia em movimento, v. 24, n. 3, 2011. Disponível em: <: / / www.scielo.br/ scielo.phppid=S010351502011000300010\&script=sci_arttext\&tl ng=es $>$ Acesso em: 05 de agosto de 2016.

PIMENTEL I.; SCHEICHER M. E. Comparação da mobilidade, força muscular e medo de cair em idosas caidoras e não caidoras. Revista Brasileira de Geriatria e Gerontologia, v.16 n.2, 2013. Disponível em: <http:/ / www.scielo.br/scielo. php?script=sci_arttext\&pid=S1809-98232013000200005> Acesso em: 17 de maio de 2017.

RECH, C. R. Antropometria para a estimativa da massa muscular. In: PETROSKI, E. L.;PIRES NETO, C. S.; GLANER, M. F.. (Org.). Biométrica Cap. 7. ed. Fontoura, Jundiaí - SP, 2010.

TIGGEMANN C. L.; Et Al. Comparação entre o treino de força tradicional e o treino de potência sobre a força muscular após destreino em mulheres idosas treinadas. Revista Brasileira de Ciência e Movimento, v.24, n.3, p. 45-52 2016.

ROGATTO, P. G.; GOBBI, S.. Efeitos da Atividade Física regular sobre parâmetros antropométricos e funcionais de mulheres jovens e idosas. Revista Brasileira de Cineantropometria \& Desempenho Humano, v.3, n. 1, 2001. Disponível em: http:/ / www.kleberpersonal.com.br/artigos/artigo_080.pdf Acesso em: 28 de agosto de 2016.

RONCATO M.; Et Al. Correlação da força e composição corporal com a capacidade funcional em mulheres idosas. Revista Brasileira de Ciência e Movimento, v. 22, n. 1, p. 130-138, 2014.

SILVA, C. M.; Et Al. Efeito do treinamento com pesos, prescrito por zona de repetições máximas, na força muscular e composição corporal em idosas. Revista Brasileira de Cineantropometria \& Desenvolvimento humano, v. 8, n. 4, p. 39-45, 2006. Disponível em: <http:/ / www.luzimarteixeira.com.br/wp-content/uploads/2011/04/sarcopenia entreinamento-com-pesos.pdf.> Acesso em: 25 de agosto de 2016.

UNICOVSKY, M. A. R.. Idoso com sarcopenia: uma abordagem do cuidado da enfermeira. Revista Brasileira de Enfermagem, v. 57, n. 3, p. 298-302, 2004. Disponível em: http://www.scielo.br/pdf/reben/v57n3/a08v57n3. Acesso em: 20 de agosto de 2016. 